\title{
Improving Genetic Knowledge Among Physicians: A Necessity in the Era of Genomic Medicine
}

Marguerite Hureaux ( $\nabla$ marguerite.hureaux@aphp.fr )

Université de Paris

\section{Sarah Watson}

PSL Research University

\section{Research Article}

Keywords: genetics, molecular biology, medical education, postgraduate

Posted Date: October 6th, 2021

DOI: https://doi.org/10.21203/rs.3.rs-948492/v1

License: (9) This work is licensed under a Creative Commons Attribution 4.0 International License. Read Full License 


\section{Abstract}

Background: Genetic analyses have experienced rapid development over the past decades. This evolution is accompanied by a considerable increase in indications in clinical practice. As a result, physicians are exposed to prescribing and understanding genetics results in their daily practice. The objective was to assess the knowledge of young physicians on new genetic techniques and their expectations concerning molecular biology education.

Methods: This study is based on the results of an anonymized questionnaire on genetics knowledge sent to young French physicians specialized in medical oncology, radiation therapy, and clinical hematology in France; and on a retrospective analysis of nonconformities to requirements of the ISO 15189 accreditation standard in genetics samples from a Parisian university hospital center.

Results: Of the 87 young physicians (median age 29, 58.6\% female) who responded to the survey, $60.3 \%$ did not differentiate several standard molecular biology techniques. Median Visual Analog Scale for theoretical knowledge of standard genetic technics was 35/100 (IQR 20-56). Median Visual Analogical Scale for genetics diagnostic and theragnostic indications knowledge were 50/100 (IQR 31.2-70) and $43 / 100$ (IQR 19.2-65), respectively. Proficiency in genetics results interpretation was assessed with a Median Visual Analog Scale of 47/100 (IQR 23.25-69). Finally, 82.4\% of young physicians believed that the current teaching of molecular biology was not suited to their current practice and $91.9 \%$ requested a specific training implementation. These results were supported by the high rate of nonconformities regarding the prescription (43\%) among the different types of nonconformities of genetics samples.

Conclusion: Medical education on new techniques of genetics by young French physicians appears to be insufficient, and a specific training should be considered. A better knowledge of this discipline is necessary in order to control the clinical, ethical and financial issues associated with the exponential use of molecular biology in medicine.

\section{Background}

Rapid development of molecular genetic techniques over the past twenty years represents a real technological revolution. These improvements lead to consider a genomic medicine approach, with personalized patient care, and open up new perspectives in predictive and preventive medicine. This technological transition is accompanied by new technological, clinical, ethical and financial challenges, involving the knowledge of these tools by prescribing physicians. In specialized centers, prescription of these tests is carried out by professionals geneticists. In certain situations, in the best interest of the patient, and / or due to local constraints of medical demography, genetics management is directly supervised by the patient's usual practitioner. Because genetic analyses have massively developed into diagnostic routines and are part of personalized medicine, it seems essential that prescribing physicians have a minimal understanding of genetics tools available, the issues they involve, and a comprehensive support for patients and their families. 
Since the development of the Sanger sequencing method, allowing the reading of around 100 bases at the end of the 1970s (1), followed by the complete sequencing of the human genome (3 billion base pairs) in 2001 (2), the advent of "next generation" sequencing (NGS: Next Generation Sequencing, or high throughput sequencing) that allows simultaneous sequencing of multiple genes, represented a major revolution in genetics and has been rapidly adopted in routine diagnosis (3). Indications have recently extended to exome sequencing (or WES for Whole Exome Sequencing, i.e. approximately 20,000 coding genes) (4), genome sequencing (or WGS for Whole Genome Sequencing, corresponding to all the DNA of an individual, non-coding and coding region) (5), and transcriptome (RNA-seq or RNA sequencing, sequencing of all messenger RNAs, i.e. genes expressed) (6). These latest technologies, WES, WGS, and RNA-seq have been available for clinical diagnosis for 1 year after specialist advice, on dedicated platforms as part of the France Médecine Génomique 2025 plan (PFMG 2025).

The development of NGS, and especially WES, RNA-seq, and WGS raises technological challenges commensurate with the quantity of data produced: 1) bioinformatics analysis, consisting in part, in alignment of the patient DNA sequence on a reference genome, and the storage of these data (1 complete genome representing approximately 3 Giga bytes (7)); 2) evaluation of the clinical value of molecular alterations identified, by "sorting" the non-pathogenic variants called polymorphisms, and possibly pathogenic variants involved in the patient's disease. In addition, these techniques are accompanied by technical constraints, specifically in somatic genetic analysis. Some approaches such as WES or RNAseq require the availability of freshly frozen tumor material, while others such as gene panel analysis can be performed on paraffin-embedded tumor material (8). These technological and technical constraints are often poorly known by prescribing clinicians and lead to unsuitable and / or non-compliant tests requests.

One of the clinical challenges lays on defining the most appropriate genetics test related to patient's phenotype. In some cases, the phenotype does not clearly guide the diagnosis, and collaboration between the biologist and the clinician is crucial to guide the genetics prescription (diagnostic or theragnostic). Another clinical challenge is the significant amount of data (WES and WGS more specifically), including variants of undetermined significance (VUS) (9), or incidental pathogenic variants that do not correspond to the patient's phenotype $(10,11)$. The ACMG (American College of Medical Genetics) clarified its recommendations for incidental findings of so-called "actionable" genes, for which prevention and / or treatment can be proposed $(11,12)$. VUS or incidental findings requires a clinical and biological confrontation, emphasizing the importance of the prescriber's knowledge and understanding of these tools. Along with technological improvement, the cost of genetic testing has drastically decreased (13). Despite this remarkable drop in cost, it remains a major public health burden due to the increase in indications (14).

Today genetics teaching in France is programmed during the first years of medical studies. Once residency status is acquired, during postgraduate medical studies, there is no specific training, while the discipline evolves very quickly. Parallelly, Health institutions are asked to conform with the accreditation process in order to monitor and improve the quality of the health care system. This process refers to the 
requirements of International System Organization IS015189 standards for medical laboratories, and includes identifying and managing nonconformities in the total test process.

The objectives of this study were to assess the knowledge in genetics and molecular biology among young prescribing physicians, and the interest of teaching this discipline during postgraduate medical studies, based on the results of a questionnaire sent to French young physicians and on the retrospective analysis of nonconformities in genetics samples from a Parisian university hospital center.

\section{Methods:}

\section{Evaluation of knowledge in molecular biology and expectations concerning the teaching of this discipline by young physicians}

An anonymized questionnaire of 31 questions was sent to French residents and young physicians in medical oncology, radiotherapy, and clinical hematology through medical associations of these specialties between March and June 2021. The questionnaire was open for responses for 3 months, from March to June 2021. The data collection platform was hosted on the SurveyMonkey site, and the survey link was accessible by computer, tablet or smartphone. The questionnaire was divided into three categories: demography of participants (7 questions), knowledge of principles, indications and technics of molecular genetics (13 questions), and training in molecular genetics (11 questions) (Supplementary Table 1). Evaluation of subjective quantitative variables was based on the use of a Visual Analog Scale (VAS) tool ranging from 0 to 100. Frequencies (percentages) and medians (interquartile ranges, IQR) were calculated to describe categorical and continuous variables.

\section{Analysis of nonconformities in genetics prescriptions}

The nonconformities to requirements of the ISO 15189 accreditation standard in genetics samples from a molecular genetics' laboratory of a Parisian university hospital center, managing oncogenetics $(40 \%$ of samples), and other constitutional hereditary disease ( $60 \%$ of samples), for samples received from January 1 to December 31,2020, were retrospectively analyzed.

\section{Results:}

\section{Evaluation of knowledge in molecular biology and expectations concerning the teaching of this discipline by young physicians}

Demographic characteristics:

The survey was sent to 500 French postgraduate medical students. A total of 87 physicians participated in the survey, of which $78(90.0 \%)$ responded the entire questionnaire (Table 1). Fifty-one participants (58.6\%) were women and the median age was 29 years (ranging from 22 to 40 years old), corresponding to the expected characteristics of French medical students. All French regions were represented, and 
Parisian residents corresponded to $36.8 \%$ of the cohort. Physicians in radiotherapy represented $42.5 \%$ of the surveyed population, followed by physicians in medical oncology (31.0\%), clinical hematology (17.2\%) then by physicians of other specialties enrolled in additional oncology course (9.3\%). $39.1 \%$ of participants were in their deepening phase of postgraduation cursus, $13.8 \%$ in their consolidation phase, 8.0\% in their initial phase, $8.0 \%$ in lay-off for research activities (Master degree or PhD students), and $17.2 \%$ in medical assistantship. A majority of participants (59.3\%) had already completed or planned to complete an additional scientific Master degree, including $46.5 \%$ in the field of biology. $10.8 \%$ of participants had already completed a 6 months internship in a medical genetics laboratory.

Table 1: Demographic characteristics of the surveyed population. 


\section{Gender}

Female

Male

Age

Median (limits)

Medical practice region

French provinces except Paris-lle-de-France

Paris-lle de France

Algeria

Unknown

\section{Medical specialty}

Medical oncology

Radiotherapy

Clinical hematology

Other specialty

Radiology

Surgery

\section{Stage* in postgraduation cursus}

Resident in initial phase

Resident in deepening phase

Resident in consolidation phase

Lay-off for research activities

Medical assistantship

Other
58,62

41,38
51

36

$29(22-40)$

$59.77 \quad 52$

$36,78 \quad 32$

$2,30 \quad 2$

1,15

1

31,03

42,53

17,24

15

4,60

4

2,30

2

2,30

2

Scientific Master degree (validated of planned) parallelly to Medical studies

Yes

59,30

No

30,23

26

Don't know

10,47 
Yes

No
10,81

89,19
8

66

* In France, the period in medical studies ranging from the final exam (postgraduation) to the medical assistantship is called medical internship or residency and is divided into 3 phases (corresponding to 4 to 5 years long): initial phase, deepening phase and consolidation phase.

Physicians knowledge in molecular biology

The second section of the questionnaire aimed to assess the theoretical knowledge of physicians regarding technical modalities, indications, results interpretation and cost of molecular biology / genetics analyses prescribed in current practice. Regarding technical modalities, $47.4 \%$ of participants did not know the difference between a Sanger sequencing analysis and a genes panel analysis by NGS, two techniques used routinely in oncology. $60.3 \%$ of participants did not know the difference between NGS and exome sequencing (Figure 1, A). Theoretical knowledge of the technical modalities of basic molecular biology tests such as PCR, targeted sequencing, or array-CGH was generally poor, with a median VAS score of 35/100 (IQR 20-56). Only one participant considered he had a perfect knowledge of these technics (Figure 1, B).

Regarding prescriptions, declared knowledge of indications to perform a genetic test for diagnostic and theragnostic purpose was also insufficient, with median VAS scores of 50/100 (IQR 31-70) and 43/100 (IQR 19-65), respectively. Proficiency in results interpretation was evaluated with a median VAS score of only 47/100 (IQR 23-69) (Figure 2). Thirteen participants (14.9\%) assessed their understanding of these technics with a VAS score less than 10/100.

Regarding the cost evaluation of genetics tests, $59 \%$ of participants admitted having no knowledge, $39.7 \%$ approximate knowledge, and only $1.3 \%$ perfect knowledge of the financial costs of molecular biology exams (Figure $3, \mathrm{~A}$ ). More specifically, only $24.4 \%$ could correctly estimate the approximate cost of a Sanger sequencing, 35\% the cost of a sequencing by genes panel in NGS, and $29.5 \%$ the cost of an exome sequencing (Figure 3, B).

Current teaching of molecular biology in medical studies and participants expectations

Regarding the current teaching of molecular biology during their medical cursus, $82.4 \%$ of participants considered that it was not suitable for their current and future practice, and only $9.5 \%$ were satisfied with the current education program (Figure 4, A). In addition, the vast majority of participants believed that the current development of their specialty requires the development of specific training for residents focused on the prescription indications of the various technics (91.9\%), and in results interpretation (95.9\%). Only $4 \%$ of participants considered that these competences were the exclusive domain of geneticists or biologists. 
Asked about their expectations regarding a possible new specific training in molecular biology, $65.7 \%$ of participants were in favor of doing a few weeks internship in a molecular biology laboratory. $52 \%$ of them even felt that this internship should be compulsory during their training (Figure 4, B), with a preference during the deepening phase (47.9\%). Participants not in favor of a compulsory internship justified their response by the limited period of postgraduate studies, or by their wish to favor their clinical experiment.

The vast majority of participants surveyed $(94.7 \%)$ requested the development of a new course module dedicated to molecular biology teaching. The preferred teaching methods was most often multimodal, combining lectures (52.0\%), e-learning (49.3\%), applied clinical cases $(42.7 \%)$ and practical work $(28 \%)$ (Figure 4, C).

\section{Analysis of nonconformities of genetics analyses prescriptions}

Of the 4,211 samples received for genetics analyses as first sample (corresponding to a new patient, therefore excluding the $2^{\text {nd }}$ samples for genetic confirmation), $18 \%$ of them presented a nonconformity upon receipt. In $56 \%$ of cases, it corresponded to a pre-analytical nonconformity (sample tube error, broken tube, identity error) and therefore concerned the sample transport chain downstream of the medical prescription (Table 2 and Figure 5, A). In 43\% of cases, it corresponded to a nonconformity of prescription, prescribed by a resident in $31 \%$ of cases, or by the senior/assistant doctor or under his/her responsibility in $69 \%$ of cases. Concerning nonconformities related to abnormal prescription, they corresponded to (1) absence of clinical information in $37 \%$ of cases, (2) absence of signed consent for genetic testing in $33 \%$ of cases, (3) abnormal or inappropriate prescription in $18 \%$ of cases, and (4) at least 2 of the above situations in $12 \%$ of cases (Figure 5, B).

Table 2: Collected nonconformities on the $1^{\text {st }}$ genetics tests requests in 2020 in a Parisian university hospital 
Total of first genetics analyses requests* in $\mathbf{2 0 2 0}$

$4211 \quad 100 \%$

Nonconformities (prescription/sample/other nonconformities)

752

$18 \%$

Sample nonconformities or pre-analytical nonconformities

421

$56 \%$

Others nonconformities

4

$<1 \%$

Prescription nonconformities

327

$43 \%$

Prescription made by medical resident

101

$31 \%$

Prescription made by senior doctor or under his/her supervision

226

$69 \%$

Details of prescription nonconformities

No consent for genetic testing form

109

$33 \%$

No clinical data information

121

$37 \%$

Abnormal prescription**

57

$17 \%$

\section{Abnormal prescription and/or no clinical data information and/or no consent for} genetic testing form

40

$12 \%$

*exclusion of the $2^{\text {nd }}$ samples for genetic confirmation; ** abnormal prescription includes obsolete or unsuitable requests (example: request for a karyotype or CGH - Comparative Genomic Hybridization for the analysis of a single gene, request for analysis of a single gene instead of a panel, request for functional tests instead of genetic analysis for metabolic diseases, request for whole genome analysis).

\section{Discussion}

Human genetics is a discipline that is currently experiencing rapid development. However, in most cases, the teaching of genetics for physicians is limited to the first years of medical studies, except in case of optional specific trainings, available from postgraduation.

The self-assessment by physicians in oncology, radiation therapy and hematology, of their knowledge in molecular biology shows that they consider it to be poor. Fewer than $10 \%$ of them believe they correctly know the technical modalities of molecular biology analyses, and their median VAS for estimating their understanding of the prescription indications and results is less than $50 / 100$. In addition, nearly $60 \%$ of them have no knowledge of the approximative cost of these exams. This lack of knowledge in genetics is accompanied by a questioning of the current methods of its teaching during postgraduate studies, with more than $80 \%$ of physician judging that current teaching is not suited to their practice and $90 \%$ willing a dedicated teaching, combining practical teaching in the laboratory and theoretical teaching with lectures and applied clinical cases. At a time of personalized medicine, this survey demonstrates the mismatch 
between the development of genetics and the training of young doctors, and therefore the urgent need for an appropriate medical education of these new technics.

The nonconformities analysis from a medical genetics laboratory of a university hospital over a period of one year confirms this lack of knowledge, by the importance of nonconformities prescription (327/4211, or $8 \%$ of the total prescriptions). These mainly correspond to a lack of clinical information (37\%), which is nevertheless essential to guide genetics prescription, that could be supplemented if necessary by analyzing new genes identified in research, illustrating the expression "from bench to bedside". In $33 \%$ of cases, nonconformities correspond to absence of consent form, which is however required by French law (Decree of 27 May 2013 regarding genetic testing for medical purposes). Finally, inappropriate prescription represents $18 \%$ of cases, directly illustrating the lack of technical knowledge in genetics of the prescriber physicians.

In addition of the negative impact for healthcare quality of the patient (wandering and delay in diagnosis or treatment), these prescription difficulties resulting in nonconformities represent a considerable work for samples reorientation and systematic prescription review. This corresponds to a significant financial cost, both in terms of medical time, and in terms of avoidable health costs in case of error or duplicated prescriptions.

Due to 1) the numerous genetic centers over the entire territory, with different or overlapping specialties and 2) the diversity of the technologies offered and the recent integration of NGS analyses for an increasing number of patients, the genetic care offer is becoming difficult to decipher for physicians. However, as radiology, well taught during medical studies, genetics and molecular biology is a medicotechnical specialty helping in the diagnosis, prognosis and monitoring of patients. Consultation between the clinician and the biologist is essential to optimize the benefit of genetic analysis.

Nevertheless, this study presents a recruitment bias as all participants are specialists in cancerology. We extrapolated the need for training in genetics to other specialties based to similar lack of specific training in these other disciplines in French medical cursus, but we cannot exclude that genetic knowledge of young physicians in other specialties, in particular pediatrics, could be better. Furthermore, the data obtained from the nonconformities collection did not allow us to study whether these were more present for oncogenetics prescriptions than for constitutional genetic prescriptions for rare diseases.

\section{Conclusion:}

This study shows the major need for evolution in the teaching of molecular biology during the postgraduate medical studies. Knowledge and understanding of these techniques by physicians are essential in the era of genomic medicine. This is essential to guarantee quality healthcare for patients, and to improve organizational, ethical and financial issues associated to genetics analyses.

\section{Abbreviations}


DNA: Desoxyribonucleic acid

RNA: Ribonucleic acid

ACMG: American College of Medical Genetics

CGH: Comparative Genomic Hybridization

COFRAC: Comité français d'accréditation, or French accreditation committee

VAS: Visual Analog Scale

IQR: interquartile range

NGS: Next Generation Sequencing

PFMG 2025: Plan France Médecine Génomique 2025, or Genomic Medicine French project 2025

RNA-seq: RNA Sequencing

VUS: Variant of Unknown Significance

WES: Whole Exome Sequencing

WGS: Whole Genome Sequencing

\section{Declarations}

ETHICS APPROVAL AND CONSENT TO PARTICIPATE STATEMENT

Non applicable.

CONSENT FOR PUBLICATION

Non applicable.

AVAILABILITY OF DATA AND MATERIAL

The authors confirm that the data supporting the findings of this study are available within the article and its supplementary materials.

CONFLICTS OF INTEREST STATEMENT

The authors declare no conflict of interest.

FUNDING STATEMENT 
The authors didn't receive any specific funding for this work.

\section{AUTHOR'S CONTRIBUTION}

$\mathrm{MH}$ and SW contributed equally to design, analyze of data, redaction and final approval of the manuscript.

\section{ACKNOWLEDGEMENTS}

The authors thank all participants that responded to the survey.

\section{References}

1. Sanger F, Nicklen S, Coulson AR: DNA sequencing with chain-terminating inhibitors. Proc Natl Acad Sci US A 74:5463-7, 1977

2. Venter JC, Adams MD, Myers EW, et al: The sequence of the human genome. Science 291:1304-51, 2001

3. Bentley DR, Balasubramanian S, Swerdlow HP, et al: Accurate whole human genome sequencing using reversible terminator chemistry. Nature 456:53-9, 2008

4. Ng SB, Turner EH, Robertson PD, et al: Targeted capture and massively parallel sequencing of 12 human exomes. Nature 461:272-6, 2009

5. Drmanac R, Sparks AB, Callow MJ, et al: Human genome sequencing using unchained base reads on self-assembling DNA nanoarrays. Science 327:78-81, 2010

6. Mortazavi A, Williams BA, McCue K, et al: Mapping and quantifying mammalian transcriptomes by RNA-Seq. Nat Methods 5:621-8, 2008

7. Treangen TJ, Salzberg SL: Repetitive DNA and next-generation sequencing: computational challenges and solutions. Nat Rev Genet 13:36-46, 2011

8. Li MM, Datto M, Duncavage EJ, et al: Standards and Guidelines for the Interpretation and Reporting of Sequence Variants in Cancer: A Joint Consensus Recommendation of the Association for Molecular Pathology, American Society of Clinical Oncology, and College of American Pathologists. J Mol Diagn 19:4-23, 2017

9. Lily Hoffman-Andrews, The known unknown: the challenges of genetic variants of uncertain significance in clinical practice, Journal of Law and the Biosciences, Volume 4, Issue 3, 648-657, 2017

10. Christenhusz GM, Devriendt K, Dierickx K: To tell or not to tell? A systematic review of ethical reflections on incidental findings arising in genetics contexts. Eur J Hum Genet 21:248-55, 2013 
11. Green RC, Berg JS, Grody WW, et al: ACMG recommendations for reporting of incidental findings in clinical exome and genome sequencing. Genet Med 15:565-74, 2013

12. Richards, S., Aziz, N., Bale, S. et al. Standards and guidelines for the interpretation of sequence variants: a joint consensus recommendation of the American College of Medical Genetics and Genomics and the Association for Molecular Pathology. Genet Med 17, 405-423 (2015).

https://doi.org/10.1038/gim.2015.30

13. Marino $P$, Touzani $R$, Borget $I$, et al: Coût du séquençage NGS à visée diagnostique en génétique somatique et constitutionnelle: estimations issues de 15 laboratoires français de génétique moléculaire des cancers. Revue d'Épidémiologie et de Santé Publique 65:S83-S84, 2017

14. Schwarze K, Buchanan J, Taylor JC, et al: Are whole-exome and whole-genome sequencing approaches cost-effective? A systematic review of the literature. Genet Med 20:1122-1130, 2018

\section{Figures}
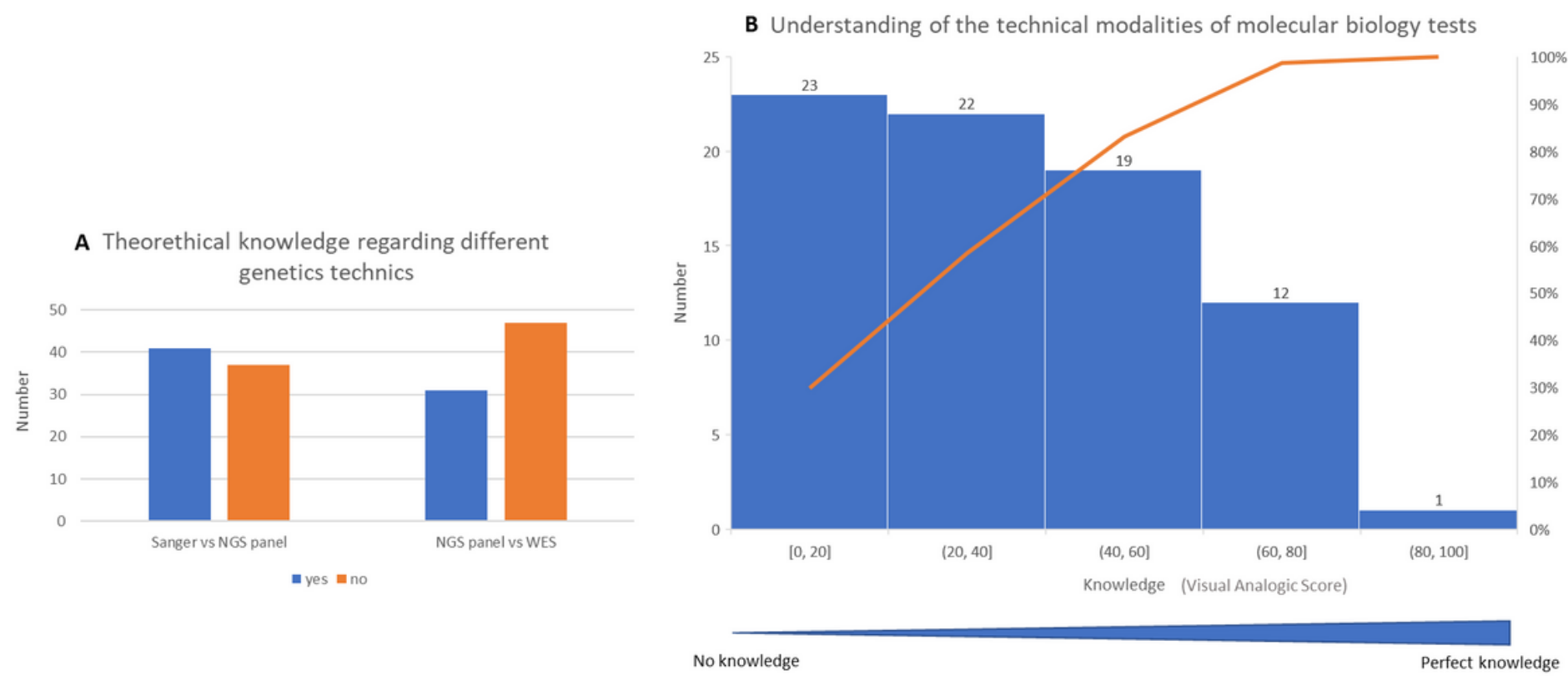

\section{Figure 1}

Theoretical knowledge (A) and understanding of the technical modalities of molecular biology tests (B) in the population surveyed. (A)Correspond to graphical representation of response to question Q8: "Do you know the difference between a Sanger sequencing of one gene and a genes panel sequencing by Next Generation Sequencing (NGS)?" and to question Q9: "Do you know the difference between a genes panel sequencing by Next Generation Sequencing (NGS) and an exome analysis?" (B)Correspond to graphical representation to question Q10: “Do you understand the technical modalities of molecular biology / genetics analyses that you prescribe, such as PCR*, targeted sequencing, MLPA*, CGH-array*, exome sequencing, qPCR*?", evaluated by a Visual Analogic Scale from 0 to 100. 


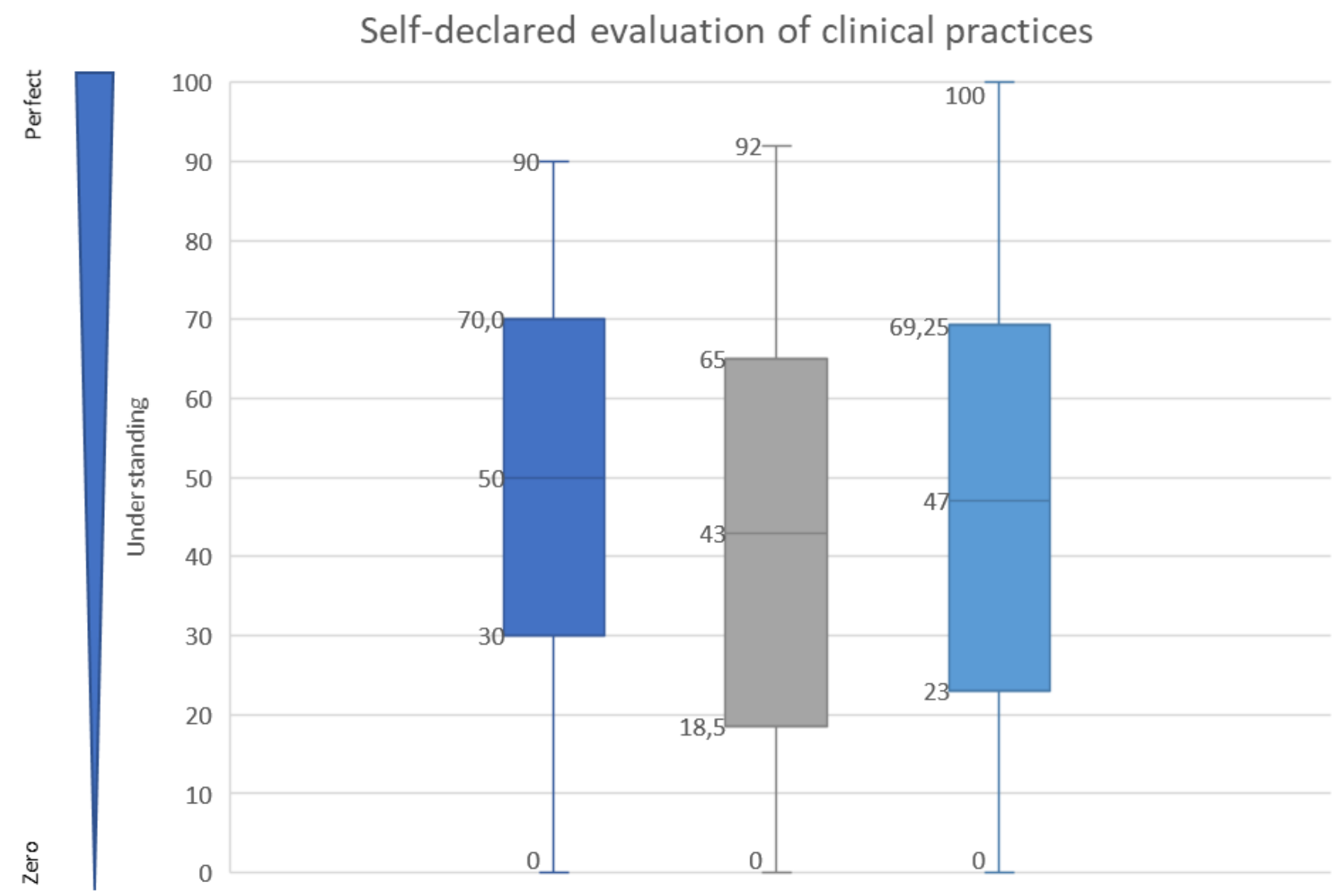

Diagnostic indications $\square$ Theragnostic indications

Results understanding

Figure 2

Self-declared evaluation of the knowledge in diagnostic and theragnostic indications to perform a genetic test, and in result interpretation understanding, based on a Visual Analogic Scale from 0 to 100. Box-plot with median, IQR, and extremes values of Visual Analogic Scale.
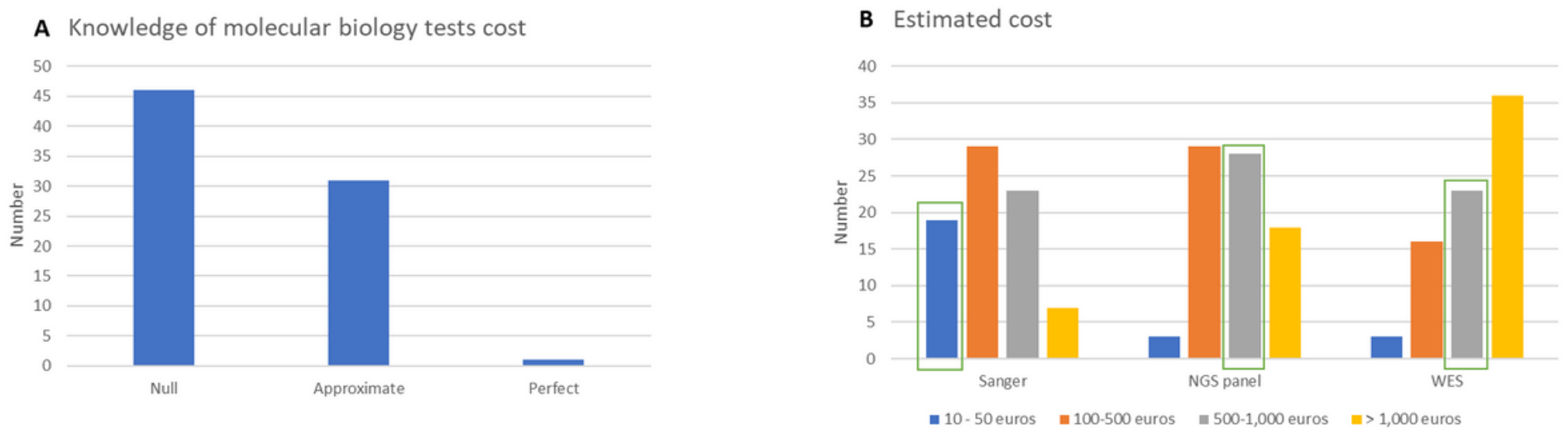

Figure 3 
Knowledge (A) of molecular biology tests cost and (B) estimation of different genetic test costs. (A)Correspond to graphical representation of response to question Q14:" Do you know how much molecular biology / genetics tests cost?" (B)Correspond to graphical representation of response to question Q15, Q16, Q17:" For example, how much would you evaluate the cost of a genetic analysis using Sanger/NGS/exome sequencing?" Current cost of these tests in France boxed in green.

A Current teaching and needs

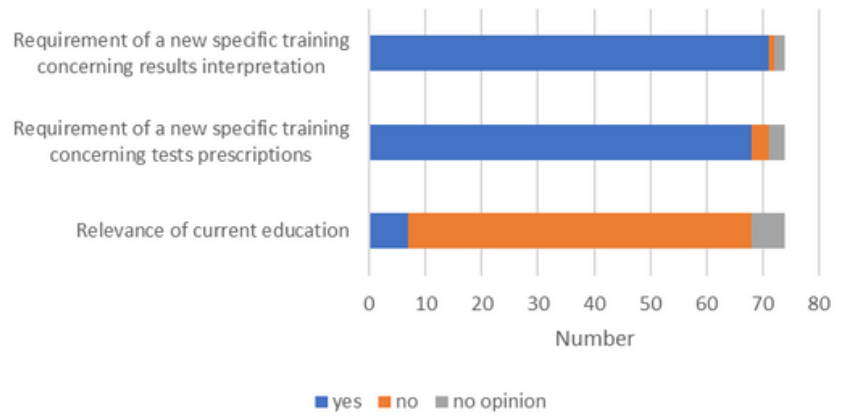

B Modalities of teaching

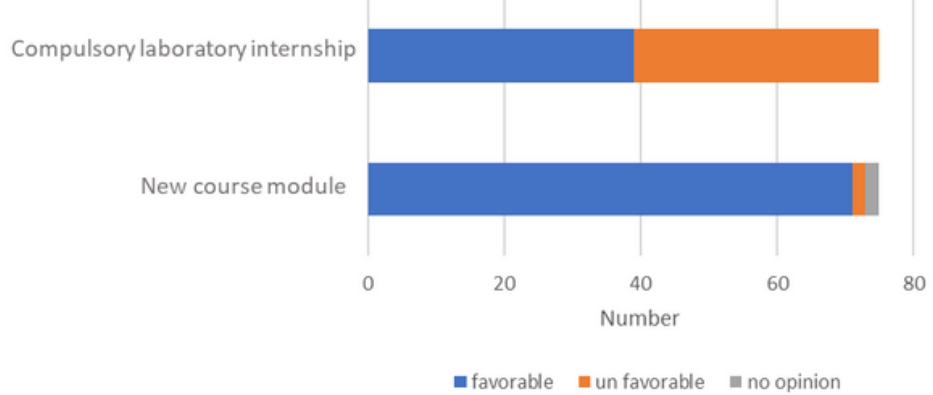

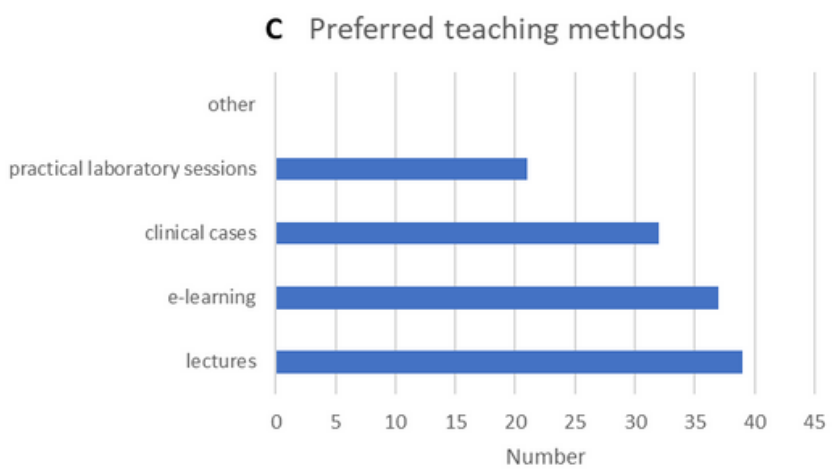

\section{Figure 4}

Current teaching of molecular biology in medical studies and participants expectations (A)Correspond to graphical representation of response to question Q21, Q22, and Q23:" Do you think that the current teaching of molecular biology / genetics as part of your training is suitable for your current and future clinical practice? Do you think that the development of your discipline requires specific training for residents to improve knowledge regarding the genetics indications of prescriptions/results interpretation?" (B)Correspond to graphical representation of response to question Q26, Q29: "Do you think that such an internship of few weeks should be compulsory during your postgraduation cursus? "and "Would you be in favor of a new training module as part of your specialty trainings on the main principles of molecular biology / genetics and its applications in your specialty? " (C) Correspond to graphical representation of response to question Q30: "If Yes to the previous question: what type of training would you prefer?" 
A Details of nonconformities in genetics analyses requests

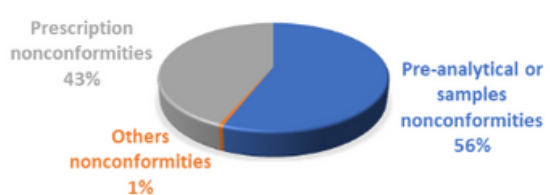

B Details of prescription nonconformities

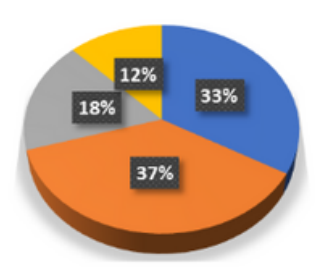

a No consent form for genetic testing

No clinical informations

abnormal prescription

Abnormal prescription* and/or no consent for genetic testing form and/or no clinical informations

\section{Figure 5}

Details of nonconformities in genetic analyses request. (A)Of the $18 \%$ total nonconformities collected, pre-analytical samples nonconformities corresponded to $56 \%$, prescription nonconformities corresponded to $43 \%$ and others nonconformities corresponded to $1 \%$. (B)Details of prescriptions nonconformities. Lack of clinical informations in $37 \%$ of prescription nonconformities, lack of consent form for genetic testing in $33 \%$, abnormal prescription in $18 \%$, and abnormal prescription and/or no consent form and/or no clinical informations in $12 \%$ of prescription nonconformities.

\section{Supplementary Files}

This is a list of supplementary files associated with this preprint. Click to download.

- SubBMCSupplementarydata.docx 